\title{
Mechanical properties of two-dimensional materials and heterostructures
}

\author{
Kai Liu ${ }^{\text {a) }}$ \\ School of Materials Science and Engineering, Tsinghua University, Beijing 100084, China \\ Junqiao $\mathrm{Wu}^{\mathrm{b})}$ \\ Department of Materials Science and Engineering, University of California, Berkeley, California 94720, USA; and \\ Materials Sciences Division, Lawrence Berkeley National Laboratory, Berkeley, California 94720, USA
}

(Received 24 August 2015; accepted 5 October 2015)

\begin{abstract}
Mechanical properties are of fundamental importance in materials science and engineering, and have been playing a great role in various materials applications in the human history. Measurements of mechanical properties of 2-dimensional (2D) materials, however, are particularly challenging. Although various types of $2 \mathrm{D}$ materials have been intensively explored in recent years, the investigation of their mechanical properties lags much behind that of other properties, leading to lots of open questions and challenges in this research field. In this review, we first introduce the nanoindentation technique with atomic force microscopy to measure the elastic properties of graphene and 2D transition metal dichalcogenides. Then we review the effect of defects on mechanical properties of 2D materials, including studies on naturally defective chemical-vapor-deposited and intentionally defective 2D materials. Lastly, we introduce a nano-electromechanical device, resonators, built on the basis of the excellent mechanical properties of $2 \mathrm{D}$ materials.
\end{abstract}

\section{INTRODUCTION}

Mechanical properties are probably the first aspect that people pay attention to in exploring a new material. Stone, bronze, and iron are the three milestone materials that appeared synchronically in ancient times. These materials were discovered, selected, and refined, mostly due to their outstanding mechanical properties, as well as cost-effective ways of production. Stone is rigid, but brittle and difficult to processing, while bronze and iron are stronger, tougher, and easier for shaping. Their utilizations have greatly changed the human history and they are used to name the ancient periods (i.e., Stone Age, Bronze Age, and Iron Age). As increasingly fast discoveries of new functional materials are made in modern times, mechanical properties are no longer the only properties that are concerned. However, they are still the key criteria in seeking super-strong or super-rigid materials for applications ranging from people's daily life to space exploration. ${ }^{1,2,3,4}$ In semiconductors, they are indispensable complements to electrical and optical properties of materials. ${ }^{5,6}$ Mechanical properties have

Contributing Editor: Jeremy T. Robinson

Address all correspondence to these authors.

a)e-mail: liuk@tsinghua.edu.cn

b)e-mail: wuj@berkeley.edu

DOI: $10.1557 / \mathrm{jmr} .2015 .324$ also played vital roles in designs of flexible, stretchable, and epidermal electronics which may potentially dominate the future electronics industry. ${ }^{7,8,9}$

Nano-science and nano-technology have attracted intensive attention for the past 30 years. ${ }^{10,11,12,13,14,15,16,17}$ In two-dimensional (2D) materials, electrons and phonons are limited in a planar dimension, and therefore many of their properties deviate from their 3D counterparts. ${ }^{16}$ Two representative examples are graphene and most transition metal dichalcogenides (TMDs) such as molybdenum disulfide $\left(\mathrm{MoS}_{2}\right)$, which, in the monolayer limit features massless Dirac fermions ${ }^{18}$ and an intrinsic direct band gap, respectively, ${ }^{19,20}$ leading to a variety of intriguing electrical and optical properties. $^{21,22,23,24,25,26,27,28}$ On the other hand, an important question arises: whether the mechanical properties of 2D materials become also different in the monolayer limit. In 3D, elastic modulus $E$ and Poisson's ratio $v$ are used to determine elastic properties of materials. $E$ is also called Young's modulus when the applied strain is uniaxial, following $E=\sigma / \varepsilon$, where $\sigma$ is stress and $\varepsilon$ is strain. Poisson's ratio $v$ is defined as the negative ratio of transverse to axial strain under uniaxial stress. Another important parameter is tensile strength, which describes the maximum tension that a material can withstand. Elastic modulus and tensile strength are scaled by the change of volumetric elastic energy, thus having a units of $\mathrm{J} / \mathrm{m}^{3}$ or $\mathrm{Pa}$. In $2 \mathrm{D}$, however, these parameters 
have to be renormalized by the planar elastic energy, leading to a units of $\mathrm{J} / \mathrm{m}^{2}$ or $\mathrm{N} / \mathrm{m}$. Although $2 \mathrm{D}$ modulus and strength are more suitable to describe 2D materials, for the purpose of comparison between 2D and 3D materials, these $2 \mathrm{D}$ parameters can be converted to $3 \mathrm{D}$ ones by dividing the 2D values with the thickness of the 2D materials. With these concepts, the conventional elastic theory can be readily applied to $2 \mathrm{D}$ systems. However, there are many new aspects of mechanical properties in 2D systems that are different from 3D systems, as we will discuss later in this review. The first step to probe mechanical properties of $2 \mathrm{D}$ systems is more technical; that is, how to accurately measure the mechanical properties of $2 \mathrm{D}$ materials.

\section{GRAPHENE-THE STRONGEST MATERIAL}

If the system is $1 \mathrm{D}$, such as a carbon nanotube or an ultra-thin silicon nanowire, measurements of Young's modulus seem to be straightforward. Stretching a nanotube or nanowire is an effective way to directly determine its mechanical properties. ${ }^{1,29,30,31}$ However, the measurements of 2D systems appear to be more difficult, because uniformly stretching a 2D membrane is challenging. A breakthrough occurred in 2008, when Lee et al. in Columbia University utilized an atomic force microscope (AFM) nanoindentation to probe suspended, circular graphene membranes. ${ }^{3}$ The AFM tip is pushing the center of the membrane (Fig. 1). When the tip radius is tiny $\left(r_{\text {tip }} \ll\right.$ $\left.r_{\text {hole }}\right)$, the load applied by the AFM tip can be considered as a point load. In a simplified continuum mechanics model, the applied load is related to the deformation geometry of the membrane, ${ }^{3,32,33}$

$$
F=\left(\sigma_{0}^{2 \mathrm{D}} \pi\right) \delta+\left(E^{2 \mathrm{D}} \frac{q^{3}}{r^{2}}\right) \delta^{3}
$$

where $F$ is the applied point load force, $\delta$ is the indentation depth at the center of the membrane, $r$ is the hole radius, $q=1 /\left(1.05-0.15 v-0.16 v^{2}\right)$ is a dimensionless constant determined by the Poisson's ratio, $v$, of the membrane, $E^{2 \mathrm{D}}$ and $\sigma_{0}^{2 \mathrm{D}}$ are the $2 \mathrm{D}$ modulus and the 2D pretension, respectively (Fig. 1).

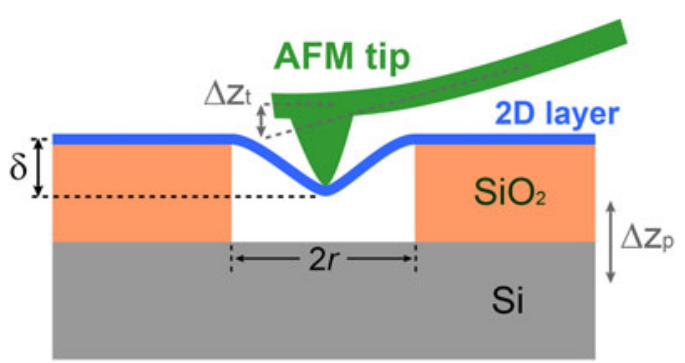

FIG. 1. Illustration of probing mechanical properties of $2 \mathrm{D}$ materials by AFM nanoindentation.
Equation (1) ignores contributions from the bending modulus because it is negligible in such an ultrathin monolayer membrane. Initially, the applied load scales linearly with the indentation depth when it is small $(F \sim \delta)$, and the coefficient is dominated by the pretention; when the indentation depth is large, the load is dominated by the stiffness of the membrane with a cubic relationship $\left(F \sim \delta^{3}\right)$. Graphene and TMDs have a lattice with 6-fold- or 3-fold-symmetry, leading to nearly isotropic mechanical properties in the plane. Thus the above model can be applied to the measurements of mechanical properties of these 2D materials.

In Eq. (1), the Poisson's ratio is an important parameter because it determines $q$ while $E^{2 \mathrm{D}}$ scales reversely with the third power of $q$. The Poisson's ratio of graphene had been actually controversial for a few years. Bulk graphite was reported to have a Poisson's ratio of 0.16 in the basal plane. ${ }^{34} \mathrm{Ab}$ initio calculation reveals an isotropic in-plane elastic response of graphene at small strains with a Poisson's ratio of 0.186 , which, at large strains, becomes anisotropic and strongly dependent on the strain values. ${ }^{34}$ On the other hand, molecular dynamic simulations show a Poisson's ratio of 0.21 for bulk graphene, and the ratio significantly depends on the size and chirality in the case of graphene nanoribbons, with a larger value in the armchair direction than in the zigzag direction. ${ }^{35}$ Despite the wide distribution of Poisson's ratio ranging from 0.16 to 0.21 , it merely introduces an error of $\sim 1 \%$ in the calculated constant $q$, or $\sim 3 \%$ for $q^{3}$, which is a negligible error for mechanical measurements.

Experimentally, monolayer graphene is mechanically exfoliated onto substrates drilled with holes with various diameters, such that the graphene membranes with desired diameters are suspended over the holes [Figs. 2(a) and 2(b)]. The indentation depth is determined by the displacement of the scanning piezo-tube of AFM $\left(\Delta z_{\mathrm{p}}\right)$ and the deflection of the AFM tip $\left(\Delta z_{\mathrm{t}}\right)$, and the applied load is obtained by multiplying the deflection of the AFM tip with its spring constant (Fig. 1). $E^{2 \mathrm{D}}$ and $\sigma_{0}^{2 \mathrm{D}}$ can be derived by a least-square fitting of the experimental forcedisplacement $[F(\delta)]$ curves with Eq. (1) [Fig. 2(c)]. By averaging over numerous times of measurements, Lee et al. obtained a mean value of $E^{2 \mathrm{D}}$ equal to $342 \mathrm{~N} / \mathrm{m}$, with a standard deviation of $30 \mathrm{~N} / \mathrm{m}$ [Fig. 2(d)]. The uncertainty of their elastic modulus measurement is not large, only $\sim 14 \%$. The derived values of $\sigma_{0}^{2 \mathrm{D}}$ ranges from 0.07 to $0.74 \mathrm{~N} / \mathrm{m}$, which are higher even than the fracture strength of many conventional materials. ${ }^{3}$ The positive pretentions may result from adhesion of graphene membrane to the vertical wall of the hole, tautly stretching the graphene membrane [Fig. 2(b)]. Assuming an effective graphene thickness of $0.335 \mathrm{~nm}$, the derived $E^{2 \mathrm{D}}$ corresponds to an ultrahigh 3D Young's modulus of $1.0 \pm 0.1 \mathrm{TPa}$, very close to the theoretical value ${ }^{36,37}$ and the in-plane Young's modulus of bulk graphite. ${ }^{34}$ 


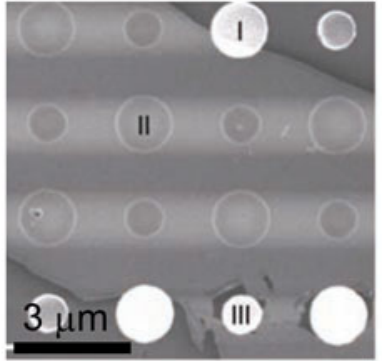

(a)

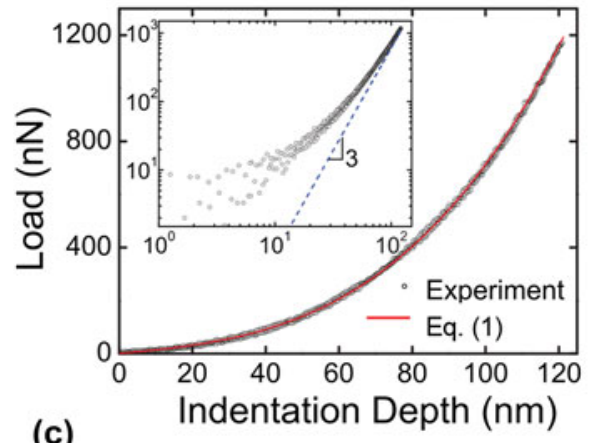

(b)

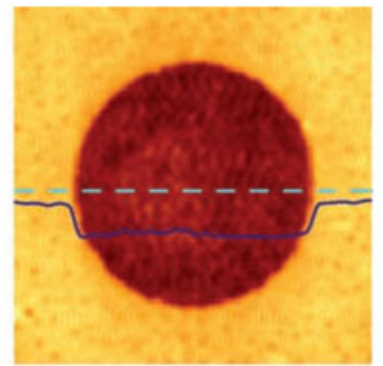

Effective Young's Modulus (TPa)

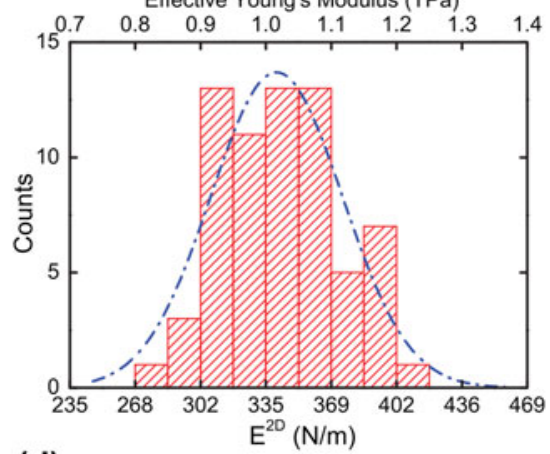

(d)

FIG. 2. Mechanical properties of exfoliated graphene: (a) scanning electron microscope image of a monolayer graphene exfoliated on a holey substrate, from Ref. 3; (b) detailed AFM image with a height profile across the dashed line, from Ref. 3; the diameter of the central hole is $1 \mu \mathrm{m}$; (c) typical experimental and fitted force-displacement curves, which reach the cubic behavior at high loads (inset), from Ref. 3; (d) statistical histogram and Gaussian distribution of measured $E^{2 \mathrm{D}}$, from Ref. 3.

This nanoindentation process can also be used to probe the tensile strength of graphene by indenting it to its breaking point. Based on a continuum model, the maximum stress for a tightly clamped, linear elastic, and circular membrane under a spherical indenter is given by ${ }^{38}$

$$
\sigma_{\mathrm{m}}^{2 \mathrm{D}}=\left(\frac{\mathrm{FE}^{2 \mathrm{D}}}{4 \pi r_{\text {tip }}}\right)^{1 / 2}
$$

where $F$ is the breaking force, and $r_{\text {tip }}$ is the tip radius. Equation (2) shows that the breaking force scales with the tip radius. For graphene, the measured data yields a mean 2D tensile strength of $55 \mathrm{~N} / \mathrm{m}$, corresponding to a 3D tensile strength of $130 \pm 10 \mathrm{GPa}$.

\section{EFFECTS OF DEFECTS}

Both the measured elastic modulus and tensile strength of graphene have the highest values among all natural materials. It arises from the extremely strong $s p^{2} \mathrm{C}-\mathrm{C}$ bonding in graphene, as well as its free from notable microscopic defects when exfoliated from high-quality bulk graphite. However, defects exist ubiquitously in all materials. In graphene, carbon atoms could be lost or displaced, generating point defects including monovacancies, di-vacancies, and Stone-Wales defects (5-7 defects). Line defects such as grain boundaries are also observed in graphene by atomically resolved transmission electron microscopy (TEM). ${ }^{39}$ The existence of these defects raises a question of how the mechanical properties of graphene are affected by defects. Theoretical simulations show that point defects weaken the elastic modulus and tensile strength of graphene, ${ }^{40,41}$ but grain boundary defects can enhance or weaken the strength of graphene depending on the tilt angles, ${ }^{42,43}$ suggesting that not only defect density affects mechanical properties of graphene, but also the arrangements and interactions between defects are important. In addition, a movement of edge dislocation pairs along the zig-zag lattice direction was observed under a low-voltage, high resolution TEM by Warner et al. ${ }^{44}$ These edge dislocations result in a substantial bond compression or elongation of $\pm 27 \%$, and their movement may contribute to the plasticity of graphene, as dislocation movement is the key mechanism for materials plastic deformation. ${ }^{44}$

In large-area graphene synthesized by chemical vapor deposition (CVD) ${ }^{45,46}$ point defects and grain boundaries are very common [Figs. 3(a) and 3(c)]. ${ }^{39,48,49,50}$ CVD graphene thus provides a good platform to test the effect of defects and grain boundaries. Early experiments show that CVD graphene has much lower Young's modulus and tensile strength than exfoliated graphene. ${ }^{51,52}$ RuizVargas et al. reported an average modulus of $55 \mathrm{~N} / \mathrm{m}$ and a breaking strength of $\sim 35 \mathrm{GPa}$, only $1 / 6$ and $1 / 4$ of the 
(a)

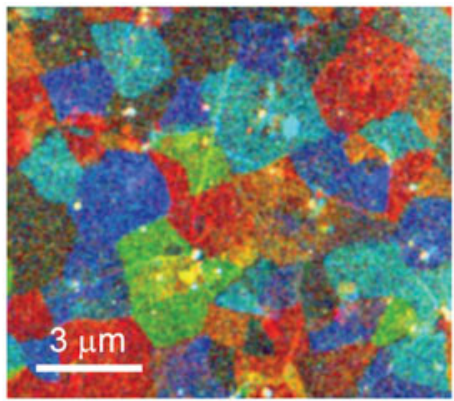

(c)
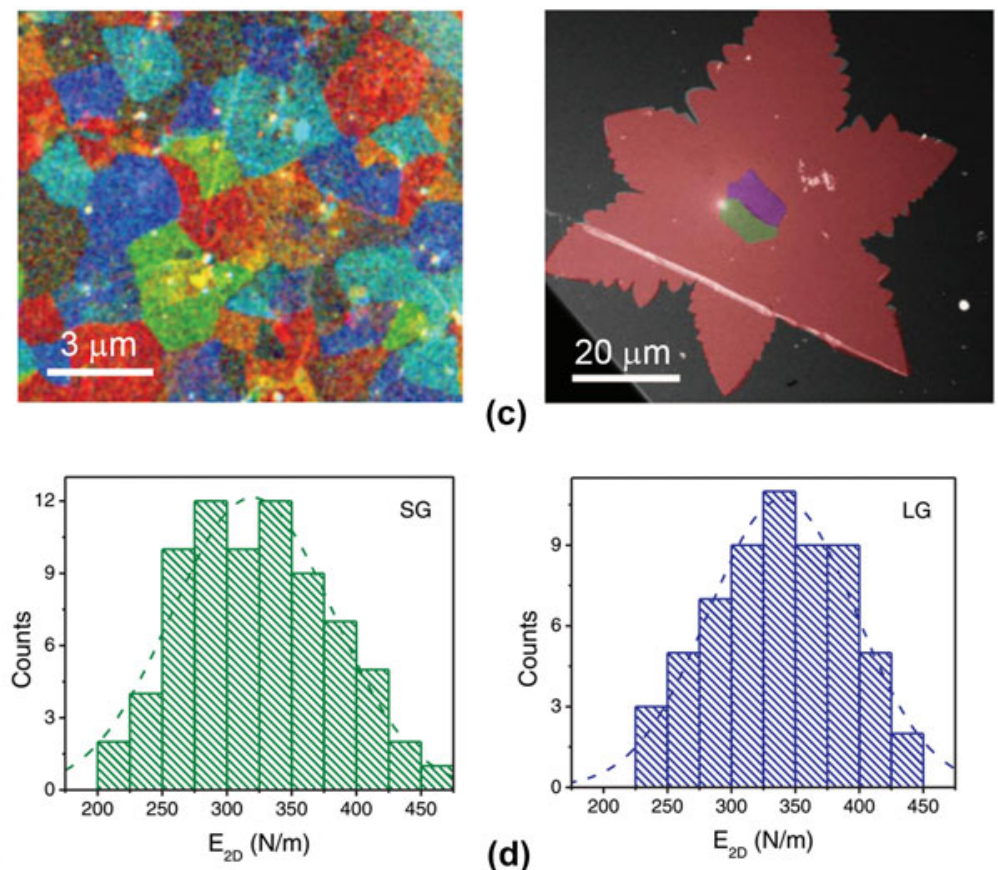

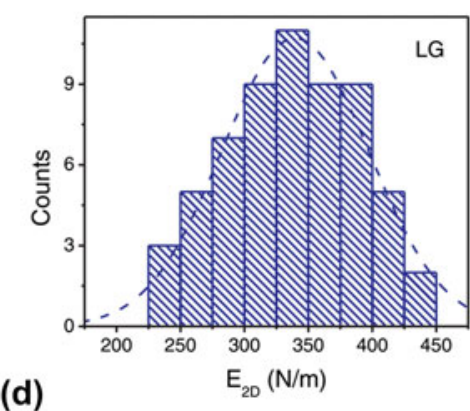

(e)
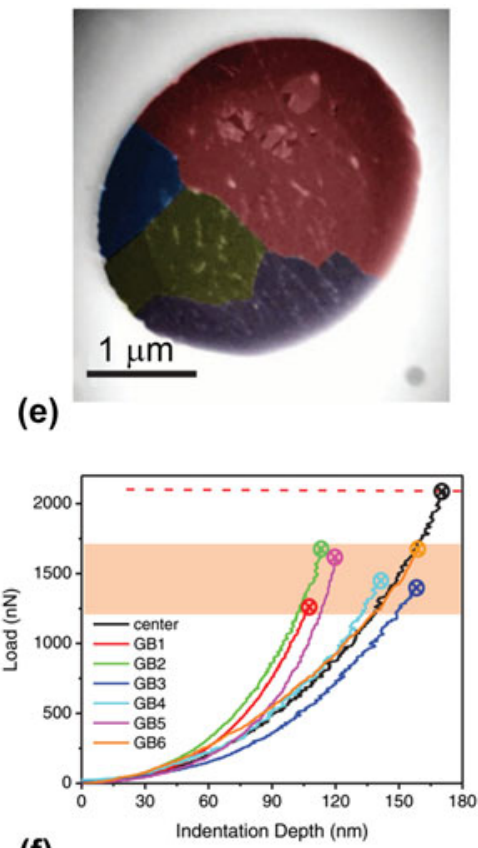

(f)

FIG. 3. Mechanical properties of CVD graphene: (a and b) a small-grain graphene: dark-field transition electron microscope (DF-TEM) image with false color (a) and histogram of its $E^{2 \mathrm{D}}$ (b), from Ref. 47; (c and d) DF-TEM images and $E^{2 \mathrm{D}}$ of a large-grain graphene, from Ref. 47; (e) bright-field TEM image of a small-grain graphene over a hole, from Ref. 47; (f) AFM indentation on different grain boundaries and on the center of the membrane as shown in (e), from Ref. 47.

values of exfoliated, single-crystal graphene, respectively. ${ }^{51}$ However, detailed studies reveal that the observed reduction in modulus and strength may originate from ripples or additional defects resulted from the transfer process. $^{47,51}$ If polymethyl methacrylate (PMMA) is coated on graphene to assist the transfer, liquid acetone is usually needed to remove the PMMA at the last step, which introduces an additional surface tension on the suspended monolayer membrane, rippling the membrane and weakening its modulus and strength.

Instead of PMMA, Lee et al. adopted polydimethylsiloxane (PDMS) as the transfer medium. They first transferred the graphene onto PDMS, and then stamped the graphene onto the target holey substrates. ${ }^{47}$ This dry transfer avoids surface tension and yields taut, suspended CVD graphene membrane, which is desired for measurements of intrinsic mechanical properties. With this effort, Lee et al. obtained an $E^{2 \mathrm{D}}$ of $328 \pm 17$ and $339 \pm 17$ $\mathrm{N} / \mathrm{m}$ for small-grain and large-grain CVD graphene [Figs. 3(a)-3(d)], both very close to the value of exfoliated, single-crystal graphene $(\sim 340 \mathrm{~N} / \mathrm{m})$. AFM indentations on grain boundaries show that facture occurs at a slightly lower load than the indentation at the center of a membrane [Figs. 3(e) and 3(f)]. The average breaking strength of graphene with small and well stitched grain, $33 \mathrm{~N} / \mathrm{m}(98.5 \mathrm{GPa})$, is slightly smaller than that of exfoliated graphene (130 GPa). ${ }^{3,47}$ These experimental and simulating results are quite surprising because a common wisdom is that a reduction of both modulus and strength is expected by defects and grain boundaries. It indicates that defects or grain boundaries play very special roles beyond normal expectation in the mechanical properties of graphene, or even other $2 \mathrm{D}$ materials.

As CVD graphene is almost as strong as exfoliated ones, the role of defects in graphene seems to be contradictory to the common sense that defects often weaken the strength of materials. One possibility is that the defect density in CVD graphene, although considerable from an electronic point of view, is still too low to affect the mechanical strength. Therefore, quantification of the dependence of mechanical properties of graphene on defect density becomes necessary. For this purpose, defects need to be intentionally introduced into graphene. Zandiatashbar et al. generated defects in graphene by a gentle oxygen plasma etching. ${ }^{53}$ Defect types and densities are estimated from the ratio of the newly appearing $D$ peak to the $G$ peak in the Raman spectra [Figs. 4(a) and 4(b)]. ${ }^{55,56}$ When the etching time is short, $s p^{3}$-type defects form, while with increasing etching time, vacancy defects appear. The 2D modulus of graphene was found to be insensitive to defects in the $s p^{3}$-type defect regime, while in the vacancy-defect regime, it drops significantly [Fig. 4(a)]. In contrast, the breaking strength (scaling with square root of breaking load) of graphene decreases monotonically with increasing defect densities induced by the oxygen plasma, although it drops only by $\sim 14 \%$ over the entire $s p^{3}$-type defect 


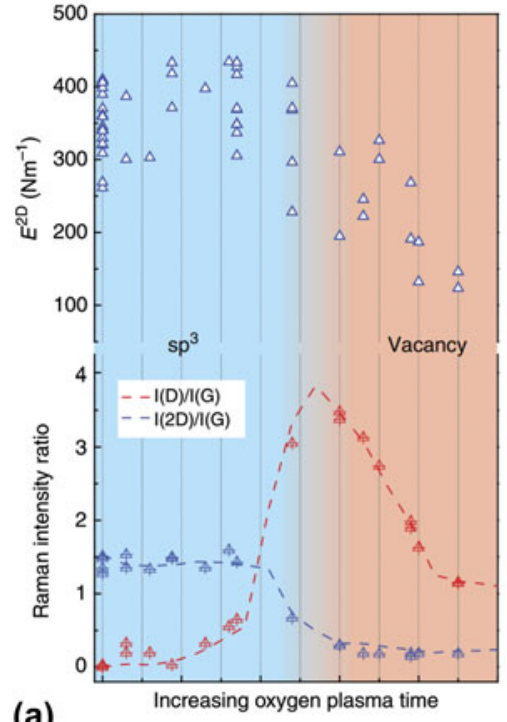
(a)

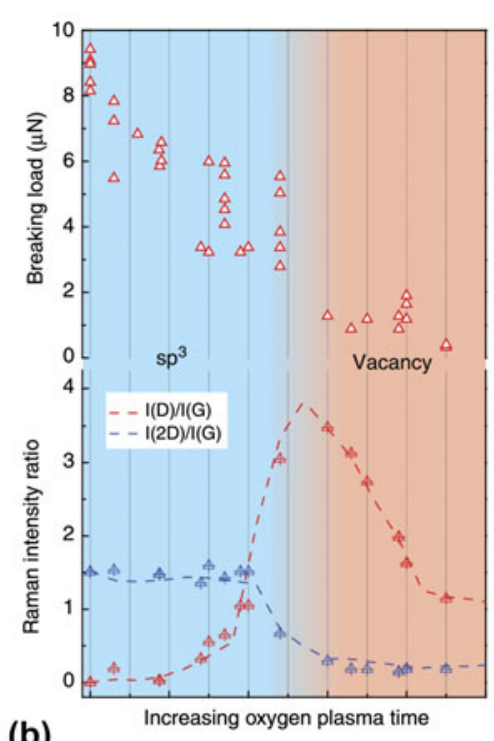

(b)
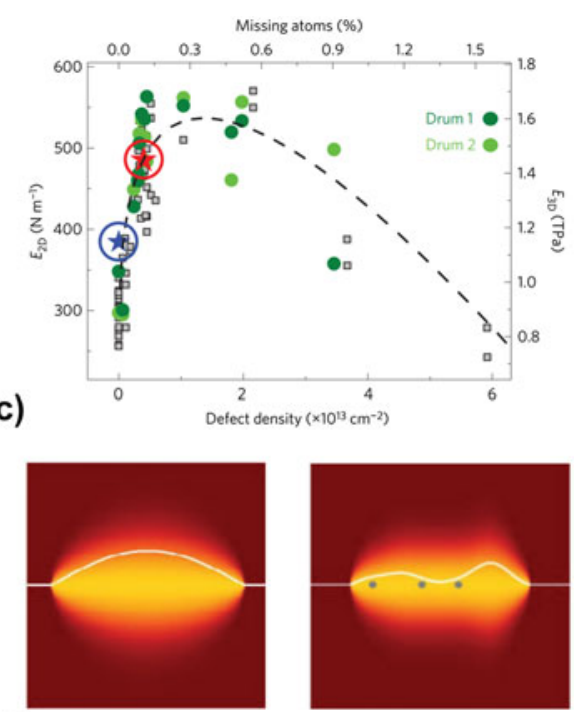

(d)

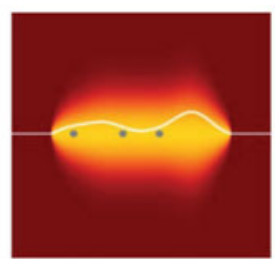

(e) etching, from Ref. 53; (c) $E^{2 \mathrm{D}}$ as a function of the density of defects introduced by $\mathrm{Ar}^{+}$irradiation, showing a non-monotonic behavior, from Ref. 54; (d) schematic pictures of thermal fluctuation of a suspended graphene membrane, and (e) of a defective graphene, from Ref. 54.

region [Fig. 4(b)]. This discovery implies that in this regime graphene may be covalently bonded with itself or to the substrate, counteracting the weakening of mechanical properties. ${ }^{53}$

Although oxygen plasma etching is an effective way to create defects, it is not a well-controlled method, because it is based on chemically reactive etching that has a fast etching rate, and tends to expand existing defects rather than generate new ones. Instead, López-Polín et al. controlled defects in monolayer graphene by a lowenergy irradiation with argon ions $\left(140 \mathrm{eV} \mathrm{Ar}{ }^{+}\right) .{ }^{54}$ This physical process creates defects uniformly and avoids the selective etching induced by oxygen plasma. With this more controlled technique, they reported an abnormal increase of $E^{2 \mathrm{D}}$ by almost a factor of 2 at a vacancy content of $0.2 \%$ [Fig. 4(c)]. Further increasing the defect density reduces $E^{2 \mathrm{D}}$. The initial increase of $E^{2 \mathrm{D}}$ was attributed to dependence of $E^{2 \mathrm{D}}$ on the momentum of flexural modes predicted for 2D membranes. In graphene, the low bending rigidity introduces significant thermal fluctuations and strong anharmonic effects, which introduces a wavevector-dependent elastic modulus. Longwavelength excitations are favorable in large and clean samples [Fig. 4(d)], but they are suppressed in defective samples [Fig. 4(e)], leading to an effective increase of the measured $E^{2 \mathrm{D}}$. This abnormal increase of $E^{2 \mathrm{D}}$ cannot be thoroughly understood from the conventional continuum mechanics, and quantum and atomic-scale theories should be also considered in accurately describing the mechanics of 2D membranes. ${ }^{54}$

In multilayer graphene, an interesting topic is the effect of defects on the interaction between graphene layers. The interlayer interaction is crucial for many applications. For example, a weak interaction is desired for applications in high-performance electrochemical devices including batteries and supercapacitors that involve atomic intercalation into the space between layers. ${ }^{57}$ In defective graphene multilayers, however, whether the interlayer interaction of graphene is enhanced or weakened has been an open question. Liu et al. used high-energy irradiation with $\alpha$ particles to introduce defects in graphene with different number of layers, and explored their elastic modulus as a function of the irradiation dose by the AFM nanoindentation method. ${ }^{58}$ They found that in irradiated multilayer graphene, carbon atoms tend to form interlayer linking around the defects and partially restore the degraded modulus. As a result of such interlayer linking, the elastic modulus of multilayer graphene shows higher resistance to radiation damage as compared to monolayers. This finding reveals the importance of interlayer linking in graphene from the mechanical perspective, and the interlayer linking may also exist in other 2D materials and heterostructures. It also sheds light for possibly engineering mechanical properties of $2 \mathrm{D}$ materials with controlled defects.

\section{MECHANICAL PROPERTIES OF 2D TMDS}

Although graphene has been proven to be the strongest material ever measured, its native zero band gap has limited its applications in semiconducting industry. ${ }^{59,60}$ As a result, TMDs, such as $\mathrm{MoS}_{2}, \mathrm{WS}_{2}$, and $\mathrm{WSe}_{2}$, have attracted much attention in recent years due to the existence of a band gap, and their crossover from indirect band gap in bulk to direct band gap in the monolayer limit. ${ }^{17}$ This opens up a wide range of 
applications for monolayer TMDs in electronics and optoelectronics. ${ }^{24,61,62}$ 2D TMDs have also been used as key components in flexible electronics, ${ }^{63,64,65,66}$ in which their mechanical properties become relevant and important. The first reports on mechanical properties of TMD layers aim at less-defective, exfoliated flakes of $\mathrm{MoS}_{2}$. Bertolazzi et al. utilized a similar AFM nanoindentation to measure the mechanical properties of exfoliated mono- and bi-layer $\mathrm{MoS}_{2}{ }^{67}$ The in-plane 2D modulus of monolayer $\mathrm{MoS}_{2}$ was determined to be $180 \pm 60 \mathrm{~N} / \mathrm{m}(270 \pm 100 \mathrm{GPa})$, and the average breaking strength $15 \pm 3 \mathrm{~N} / \mathrm{m}(23 \mathrm{GPa})$, which are several times lower than monolayer graphene but still much stronger than steel. The pretention lies between 0.02 and $0.1 \mathrm{~N} / \mathrm{m}$. In contrast, bilayer $\mathrm{MoS}_{2}$ has a $2 \mathrm{D}$ modulus of $260 \pm 70 \mathrm{~N} / \mathrm{m}$, corresponding to a lower 3D modulus of $200 \pm 60 \mathrm{GPa}$, which is attributed to defects or interlayer sliding. ${ }^{67}$ On the other hand, CastellanosGomez et al. studied the elastic properties of suspended, thick $\mathrm{MoS}_{2}$ sheets with 5-25 layers. ${ }^{68}$ In their work, the bending rigidity has to be considered and one more linear term, $\left[\frac{4 \pi \mathrm{E}^{2 \mathrm{D}}}{3\left(1-\mathrm{v}^{2}\right)} \cdot \frac{t^{2}}{r^{2}}\right] \delta$, needs to be added into Eq. (1), where $t$ and $r$ are the thickness and the radius of membrane, respectively. Despite this additional term, the modulus and the pretension can be similarly derived by fitting to the experimental force-displacement curves. They obtained a mean Young's modulus of $330 \pm 70 \mathrm{GPa}$, and a pretention of $0.13 \pm 0.10 \mathrm{~N} / \mathrm{m} .{ }^{68}$ Both values are higher than the results obtained by Bertolazzi et al., ${ }^{67}$ and both modulus values obtained by these two groups are higher than bulk $\mathrm{MoS}_{2}(\sim 240 \mathrm{GPa}){ }^{69}$

A similar question to that in graphene appears here in 2D TMDs, that is, whether CVD samples have similar mechanical properties as exfoliated samples. CVD is a widely applied method to grow large-area materials in cost-effective ways. But CVD samples are usually more defective compared to single crystals. So far, people have developed CVD methods to synthesize largearea, ${ }^{70,71,72,73}$ or even wafer-scale, ${ }^{74}$ 2D TMDs such as $\mathrm{MoS}_{2}$. The as-grown samples consist of isolated triangles of monolayer crystals at positions on the substrate slightly far away from the precursors, and merged continuous film closer to the precursors [Figs. 5(a)-5(c)]. Liu et al. transferred CVD $\mathrm{MoS}_{2}$ and $\mathrm{WS}_{2}$ with a similar PDMS stamping process onto holey substrates [Figs. 5(d) and $5(\mathrm{f})],{ }^{75}$ as Lee et al. did in CVD graphene, ${ }^{47}$ to avoid ripples that complicated the measurements of mechanical properties. They also employ the AFM nanoindentation to probe the modulus of monolayer CVD $\mathrm{MoS}_{2}$ and $\mathrm{WS}_{2}$, finding values of $171 \pm 11$ $(264 \mathrm{GPa})$ and $177 \pm 12 \mathrm{~N} / \mathrm{m}(272 \mathrm{GPa})$, respectively [Figs. 5(f) and 5(g)]. The nearly identical values between $\mathrm{MoS}_{2}$ and $\mathrm{WS}_{2}$ originate from their similar lattice constants and bonding energies. First-principles calculations predict a $2 \mathrm{D}$ modulus of $123 \mathrm{~N} / \mathrm{m}$ for

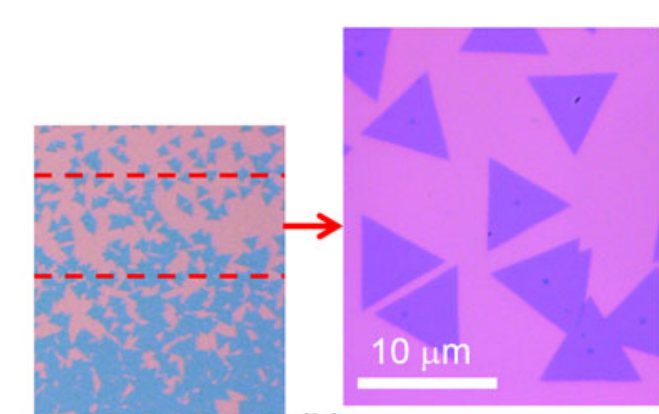

(b)

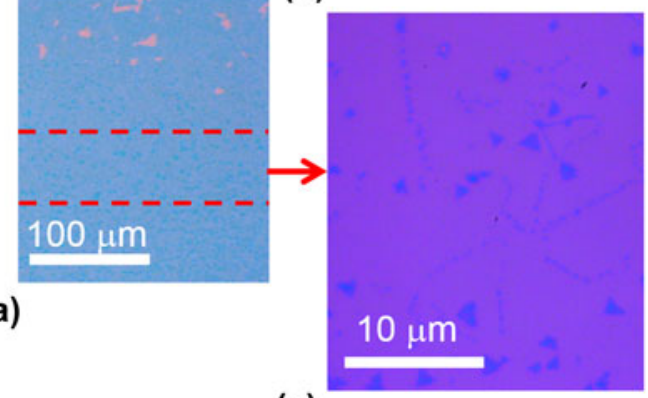

(c)

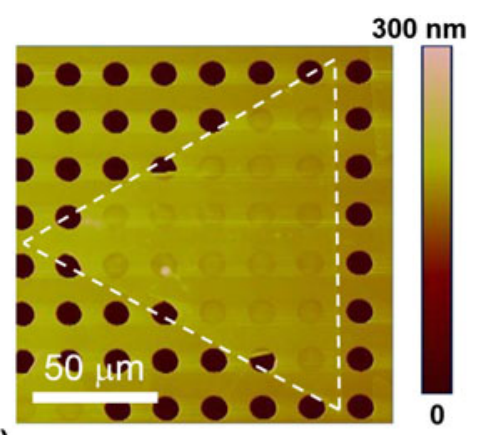

(d)

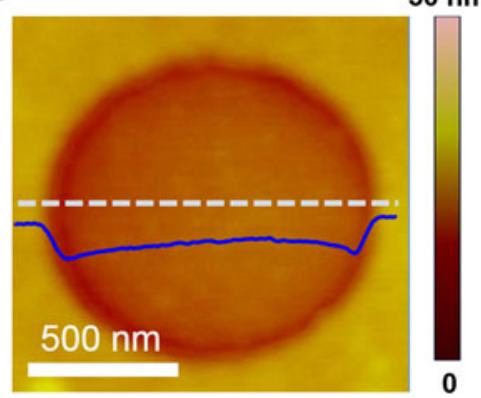

(e)

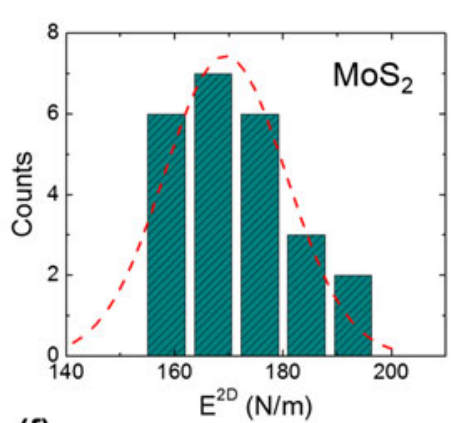

(f)

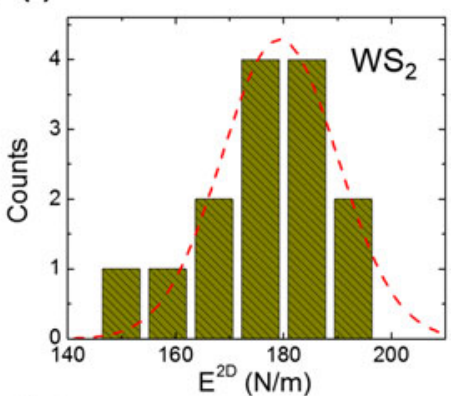

(g)

FIG. 5. Measurements of CVD $\mathrm{MoS}_{2}$ and $\mathrm{WS}_{2}$ monolayers: (a-c) as-grown CVD $\mathrm{MoS}_{2}$ samples (a), consisting of isolated-triangle part (b) and continuous film (c), from Ref. 75; (d) AFM image of an entire triangle transferred onto a holey substrate, from Ref. 75; (e) AFM image of a suspended membrane over a hole, from Ref. 75; (f and g) histograms of $E^{2 \mathrm{D}}$ for $\mathrm{MoS}_{2}$ and $\mathrm{WS}_{2}$, and the corresponding Guassian distribution, from Ref. 75. 
$\mathrm{MoS}_{2}$ and $137 \mathrm{~N} / \mathrm{m}$ for $\mathrm{WS}_{2}$, both lower than experimental values, which is consistent with the fact that the used DFT-GGA calculation usually underestimates the bulk modulus of semiconductor materials. ${ }^{76}$ Note that Liu et al. only measure the membranes with a single grain, so the effect of grain boundaries is not probed here. All of the elastic moduli and break strengths for 2D materials ever measured are listed in Table I. The modulus of CVD $\operatorname{MoS}_{2}(\sim 170 \mathrm{~N} / \mathrm{m})$ is only $\sim 5 \%$ lower than that of exfoliated $\mathrm{MoS}_{2}$ $(\sim 180 \mathrm{~N} / \mathrm{m}),{ }^{67}$ similar to the case in CVD graphene versus exfoliated graphene. It may imply that point defects in CVD samples do not significantly affect the mechanical properties of CVD samples. However, there is no simple way to quantify the defects in $2 \mathrm{D}$ TMDs, because unlike graphene, 2D TMDs have no defect-related Raman peaks. This leaves an interesting topic for quantifying the relationship between mechanical properties and defects density in 2D TMDs.

TABLE I. Elastic properties of 2D materials ever measured.

\begin{tabular}{|c|c|c|c|}
\hline Materials & $\begin{array}{l}\text { 3D Young's } \\
\text { modulus (GPa) }\end{array}$ & $\begin{array}{c}\text { Tensile } \\
\text { strength (GPa) }\end{array}$ & References \\
\hline Graphite & 1000 & & 34 \\
\hline Exfoliated graphene (1L) & 1000 & 130 & 3 \\
\hline CVD graphene (1L) & 979 & 98.5 & 47 \\
\hline Bulk $\mathrm{MoS}_{2}$ & 240 & $\ldots$ & 69 \\
\hline Exfoliated $\mathrm{MoS}_{2}(1 \mathrm{~L})$ & 270 & 23 & 67 \\
\hline Exfoliated $\mathrm{MoS}_{2}(\mathrm{ML})$ & 330 & $\ldots$ & 68 \\
\hline $\mathrm{CVD} \mathrm{MoS}$ (1L) & 264 & $\ldots$ & 75 \\
\hline $\mathrm{CVD} \mathrm{WS}_{2}(1 \mathrm{~L})$ & 272 & $\ldots$ & 75 \\
\hline
\end{tabular}

Note: "1L" and "ML" indicate monolayer and multilayer, respectively.

\section{2D VAN DER WAALS HETEROSTRUCTURES}

Stacking a 2D sheet onto another by van der Waals interactions and forming a heterostructure may be the most promising advantage of $2 \mathrm{D}$ materials in electronic applications (Fig. 6). ${ }^{60}$ This provides a simple, controllable way to build up heterostructures with desired band alignments. ${ }^{77}$ Their great potentials in optoelectronics and multi-junction solar cells have aroused lots of studies in recent years. ${ }^{78,79,80,81}$ Interlayer coupling has been found to play a great role in the performance of $2 \mathrm{D}$ heterostructure-based devices, and has been investigated intensively from the electrical or optical perspective. ${ }^{82,83,84,85,86}$ However, mechanical probing of the heterostructures is still lacking.

There are some ways to investigate the interlayer coupling from a mechanical point of view. It seems to be not so difficult in 2D homostructures, such as multilayer graphene or bulk graphite. Tan et al. measured the interlayer shear Raman modes in low energies in multilayer graphene. ${ }^{87}$ The corresponding Raman peaks shift from bilayer graphene $\left(\sim 31 \mathrm{~cm}^{-1}\right)$ to bulk graphite $\left(\sim 43 \mathrm{~cm}^{-1}\right)$, suggesting that the peaks are related to interlayer interaction. With a deeper analysis, the shear modulus across the layer-layer interface is derived to be $4.3 \mathrm{GPa}$ in graphite. ${ }^{88}$ This indirect method could be also applied to 2D heterostructures. Koren et al. used an AFM to apply lateral shear force on predesigned highly oriented pyrolytic graphite (HOPG) patterns. ${ }^{89}$ This induces a shear gliding along a random single basal plane in the HOPG patterns, from which the lateral shear force and the friction force can be obtained. The interface adhesion energy is measured to be $0.227 \pm 0.005 \mathrm{~J} / \mathrm{m}^{2}$, which is agreement with theoretical models. It is a direct
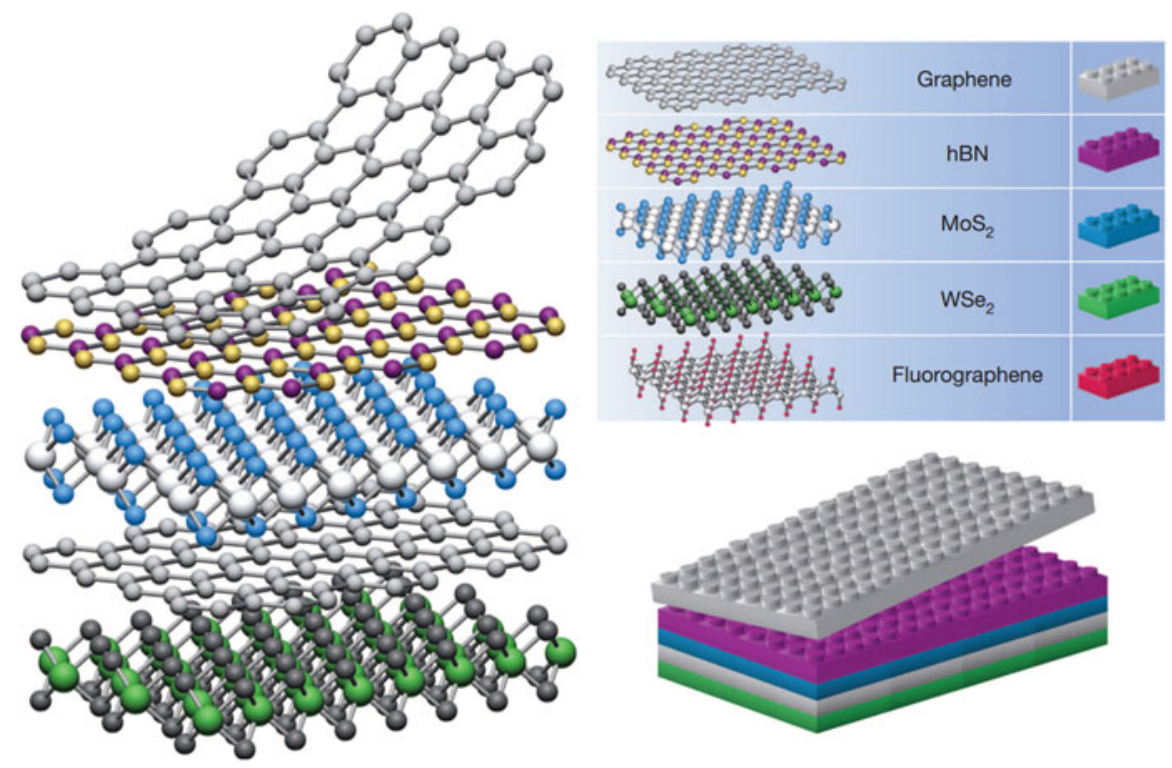

FIG. 6. Schematic of van der Waals heterostructures, from Ref. 60. 
method to quantify the interlayer interaction in 2D homostructures, but it is not very suitable to probe heterostructures, because thick 2D materials are required in their method but it is difficult to form thick heterostructures by stacking monolayers, and the basal plane where the shear gliding occurs is difficult to be well controlled at the hetero-interface.

Mechanically probing the interlayer interaction in 2D heterostructures is still a challenge. With a purpose of attempting to address this, Liu et al. probed the elastic modulus of 2D bilayer heterostructures by the wellestablished nanoindentation process, along with their measurements of 2D TMD monolayers. ${ }^{75}$ They found that the measured $E^{2 \mathrm{D}}$ of bilayer heterostructures are always lower than the sum of $E^{2 \mathrm{D}}$ of each layer, implying an interlayer sliding that depends on the interlayer interaction [Fig. 7(a)]. In their experiments, the bilayer is suspended over a circular hole. The bottom layer in direct contact with the substrate is firmly clamped onto the substrate. In the extreme case of a very strong interlayer interaction, there is no interlayer sliding allowed, such that both layers contribute to the 2D modulus measured. In the opposite extreme, however, when the top layer is completely free to slide against the bottom layer, the measured modulus of the bilayer is solely given by the bottom layer that is clamped onto the substrate. With these considerations, they describe the measured modulus of a $2 \mathrm{D}$ bilayer with a phenomenological equation, $E_{\mathrm{t}}^{2 \mathrm{D}}=E_{\mathrm{bot}}^{2 \mathrm{D}}+\alpha \cdot E_{\mathrm{top}}^{2 \mathrm{D}}$, where $E_{\mathrm{t}}^{2 \mathrm{D}}, E_{\mathrm{bot}}^{2 \mathrm{D}}$, and $E_{\text {top }}^{2 \mathrm{D}}$ are the $2 \mathrm{D}$ modulus of the bilayer, the bottom layer, and the top layer, respectively. $\alpha$ is named "interaction coefficient," which ranges from 0 to 1 , qualitatively gauging the strength of the interlayer interaction. From their measurements, $\alpha$ is 0.80 in $\mathrm{MoS}_{2} / \mathrm{WS}_{2}$ heterostructure, comparable to 0.75 in $\mathrm{MoS}_{2} / \mathrm{MoS}_{2}$ bilayer, but it is only 0.69 in $\mathrm{MoS}_{2}$ /graphene heterostructure [Fig. 7(b)]. Although this method is not able to directly quantify the interlayer interaction, it provides a relatively simple way to explore the interlayer interaction in 2D heterostructures. Further investigation of $\alpha$ may be useful for extracting more intrinsic physical parameters, such as adhesion energy and friction coefficient, in bilayer heterostructures.

\section{RESONATORS BASED ON GRAPHENE AND $\mathrm{MoS}_{2}$}

2D materials are ultrathin and atomically flat, providing perfect candidates for planar nanoscale mechanical devices. Resonator is an electromechanical device that vibrates in response to an external applied force. ${ }^{90}$ As their resonant vibrations are sensitive to external environment, resonators can be used as sensors for mass, force, and charge, etc. The first prototype of graphene resonator was developed by Bunch et al. at Cornell University. ${ }^{91}$ They mechanically exfoliated graphene nanosheets on predefined trenches that are etched into a $\mathrm{SiO}_{2}$ surface. The resultant resonators are a doubly clamped, ultrathin, and microscale ribbon-like device [Fig. 8(a)]. These resonators are actuated by an electrical modulation that is realized by a time-varying radio frequency (rf) voltage on top of a constant voltage, or an optical modulation by using a modulated, focused diode laser at frequency $f$. The vibration of the resonators is monitored by the reflected light intensity from another laser beam with a photodiode. Multiple resonances are observed in graphene resonators, but the most prominent one at the lowest frequency is attributed to the fundamental vibrational mode, $f_{0}$, which is related to the tension $T$ and determined by ${ }^{91}$

$$
f_{0}=\left\{\left[A(E / \rho)^{1 / 2} t / L^{2}\right]^{2}+A^{2} 0.57 T / \rho L^{2} w t\right\}^{1 / 2},
$$

where $A$ is the clamping coefficient (1.03 for doubly clamped ribbons and 0.162 for cantilevers), $E$ is the
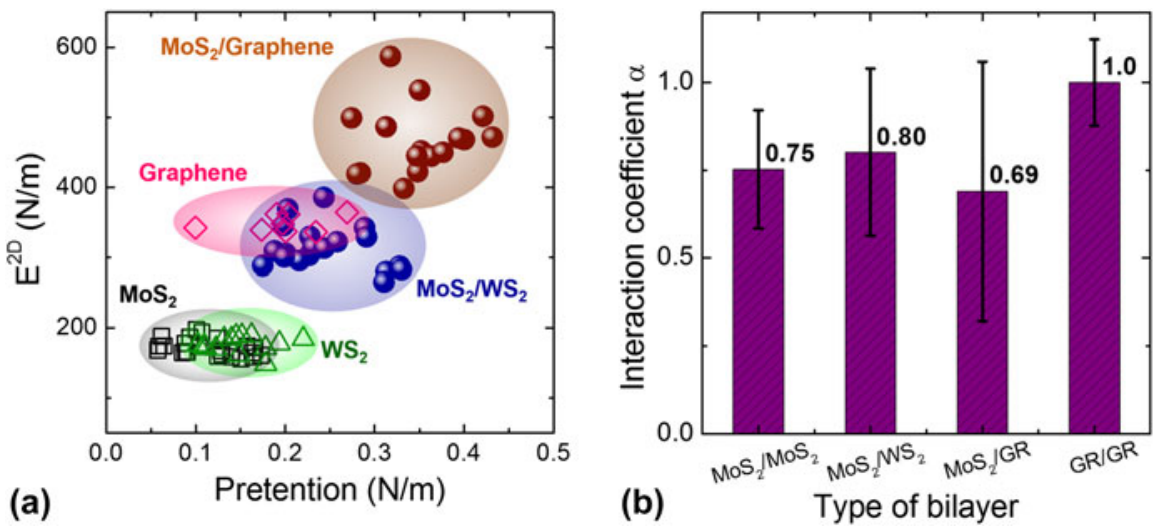

FIG. 7. Elastic properties of 2D heterostructures: (a) measured $E^{2 \mathrm{D}}$ and pretensions of various $2 \mathrm{D}$ monolayers and heterostructures, from Ref. 75 ; (b) defined interaction coefficients for different types of bilayers, from Ref. 75. 


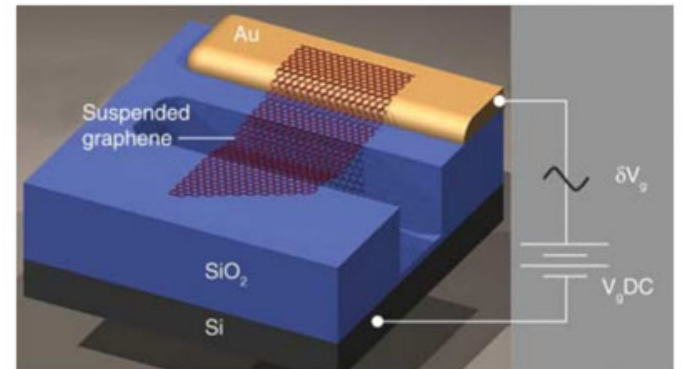

(a)

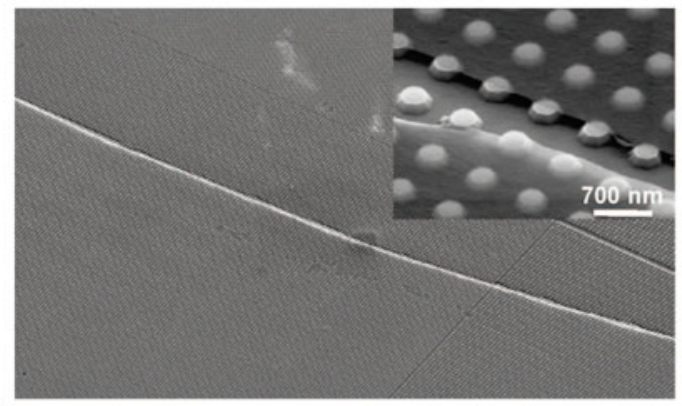

(d)

(e)

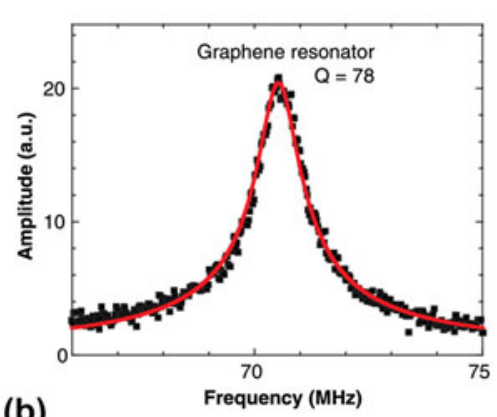

(b)

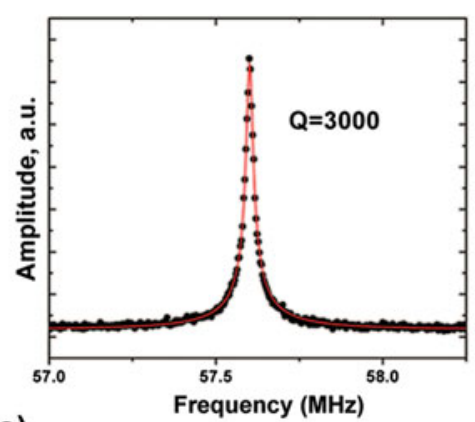

Frequency $(\mathrm{MHz})$

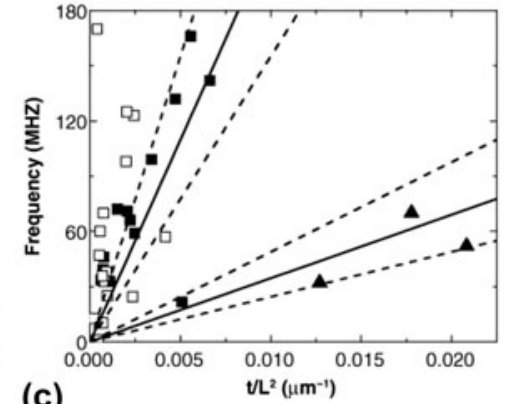

(c)

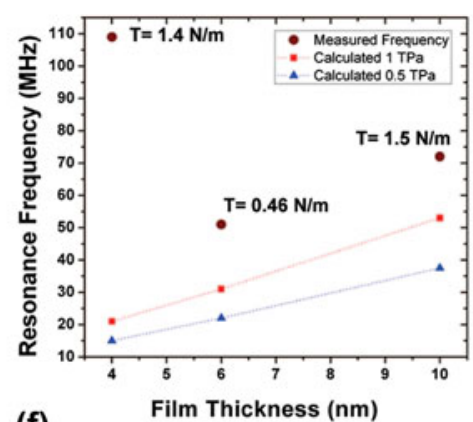

(f)

FIG. 8. Graphene-based resonators: (a) schematic of a graphene resonator, from Ref. 91; (b) frequency response of a graphene resonator, with a Lorentzian fit, from Ref. 91; (c) scaling the frequency with $t / L^{2}$, from Ref. 91; (d) large-area rGO film transferred on a pre-patterned substrate; rGO resonators are created by a similar transfer process, from Ref. 92; (e) frequency response of a rGO resonator, from Ref. 92; (f) experimental resonance frequencies at different film thicknesses and tensions, as well as theoretical values plotted for flat circular membranes with two different moduli, from Ref. 92.

Young's modulus, $\rho$ is the mass density, $t, w$, and $L$ are the thickness, width, and length of the ribbon. When the tension $T$ is small, the second term in Eq. (3) can be ignored and $f_{0}$ scales with $t / L^{2}$. Their results show that $f_{0}$ varies from 1 to $170 \mathrm{MHz}$, and the quality factor $Q$ from 20 to 850 [Fig. 8(b)]. All of the data are plotted in Fig. 8(c), where the solid line corresponds to $E=1 \mathrm{TPa}$, and dashed lines to the estimated lowest modulus $(0.5 \mathrm{TPa})$ and the highest $(2 \mathrm{TPa})$. For the resonators with $t>7 \mathrm{~nm}$, almost all data points lie between the two dashed lines, giving the resonators with the highest modulus to date. For the resonators with $t<7 \mathrm{~nm}$, however, most of their resonant frequency are higher than predicted, which indicates that the tension is not negligible in this case. ${ }^{91}$

Ultrahigh Young's modulus, extremely low mass, and large surface area make graphene resonators an ideal candidate for ultrasensitive sensors. Chen et al. developed an all-electrical, high-frequency mixing method for the actuation and detection of graphene resonances as mass sensors. ${ }^{93}$ In their method, an rf gate voltage $\delta V_{\mathrm{g}}^{f}$ at a frequency of $f$ is applied on top of a dc gate voltage $V_{\mathrm{g}}$ to drive the motion, and another rf voltage with a small offset frequency $f+\Delta f$ is applied to the source. The mixed-down current $\mathrm{I}^{\Delta f}$ at the frequency difference $\Delta f$ is measured to detect the motion because the conductance of monolayer graphene is gate responsive. Their devices also work in the megahertz range. They found that the interactions between adsorbates and the graphene sheet must be considered because adsorbates impart tension to the graphene. As a mass sensor, their best sample shows a sensitivity of $\sim 2 \mathrm{zg}$. The quality factor of the graphene resonator increases with lowering temperature, reaching 14,000 at $5 \mathrm{~K}$, in contrast to a value of 125 at room temperature.

Although resonators based on exfoliated graphene are suitable for fundamental studies, large-area devices are much desired in realistic applications. Robinson et al. fabricated wafer-scale, few-monolayer reduced graphene oxide (rGO) films and then assembled them to mechanical resonators [Fig. 8(d)]. ${ }^{92,94}$ They utilized a wellestablished technique of laser interferometry to detect the resonance. The resonant frequencies of their devices lie in the megahertz range, and their modulus is moderately high $(\sim 185 \mathrm{GPa})$, but their quality factors and figures of merit $(f \times Q)$ can reach up to 4000 and more than $10^{11} \mathrm{~Hz}$ at room temperature [Fig. 8(e)], ${ }^{92}$ which are much higher than the values of pure graphene resonators aforementioned. The experimental resonance frequencies are much higher than the theoretical values of relaxed films, suggesting considerable tensions that contribute to the high $Q$ values [Fig. 8(f)]. This work not only opens up a way to produce large-area, high Young's modulus, low-density mechanical devices based on rGO films, but 
also reveals the importance of imposed tension for the $Q$ factor of graphene resonators. The reason for the low $Q$ factors in graphene resonators has been heavily debated. As the $Q$ factor strongly depends on temperature, ${ }^{93}$ tension, ${ }^{92}$ and damping, ${ }^{95}$ it implies that thermal fluctuation out of the plane in such atomically thin material might play a role. However, more experiments and simulations are needed for a full understanding, leaving an interesting topic in this research field.

Besides graphene, other 2D materials can also be adopted as the working component of mechanical resonators. $\mathrm{MoS}_{2}$ is good candidate for this purpose because it is the mostly studied 2D TMDs with excellent mechanical properties. As discussed above, monolayer or thin-layer $\mathrm{MoS}_{2}$ has a Young's modulus of 270-330 GPa, which reaches almost $1 / 3$ of the value of graphene. ${ }^{67,68,75}$ Lee et al. reported multilayer $\mathrm{MoS}_{2}$-based nanomechanical resonators with a minimum thickness of $6 \mathrm{~nm}$ (9 layers). ${ }^{96}$ Their devices exhibit a fundamental resonance frequency $f \sim 60 \mathrm{MHz}$ and a figure of merit $f \times Q \sim 2 \times 10^{10} \mathrm{~Hz}$, both are actually lower than graphene resonators. Castellanos-Gomez et al. studied the monolayer $\mathrm{MoS}_{2}$ mechanical resonator. ${ }^{97}$ They report a similar range of values, $f$ ranging from 10 to $30 \mathrm{MHz}$ and quality factor being $\sim 55$ at room temperature. The mechanics is found to be tension dominated for monolayer $\mathrm{MoS}_{2}$ resonators, but bending rigidity dominated for multilayer $\mathrm{MoS}_{2}$ devices, a similar conclusion drawn in graphene devices. As 2D TMDs has an intrinsic direct band gap that is tunable by strain, these findings on $\mathrm{MoS}_{2}$ may illuminate novel devices that combine nanomechanical and optoelectronic properties of 2D TMDs.

\section{SUMMARY}

We have summarized recent progresses on the studies of mechanical properties of 2D materials, 2D heterostructures, and their applications as a particular type of mechanical devices, resonators. We have also discussed the effect of defects on mechanical properties of graphene. Together with electrical, optical, and thermal properties, mechanical properties are among the most important and interesting properties of $2 \mathrm{D}$ materials. Graphene has been proven to be the strongest 2D material in nature. Other 2D materials, such as $\mathrm{MoS}_{2}$, also have competent mechanical properties compared to conventional materials such as steel. Their large surface area, high Young's modulus, and extremely low mass have yielded high-performance resonators that are ideal for ultrasensitive mass, force, and charge sensors. The mechanical probing of $2 \mathrm{D}$ heterostructures provides valuable information for understanding the effect of interlayer interactions. The progresses are remarkable in both measurements and applications. However, there are still open questions and challenges remaining in this research field.
Although more and more types of 2D monolayers are explored, their mechanical properties are relatively less explored, lagging behind studies of their electrical, optical, or even thermal properties. So far, mechanical properties are only investigated for several 2D monolayers including graphene, $\mathrm{MoS}_{2}$, and $\mathrm{WS}_{2}$, and the atlas of mechanics has much empty phase space for a wide range of 2D materials. Filling-up of the atlas is technically feasible, and it is particularly important for the future design of flexible devices based on various 2D materials. Especially, investigation of the anisotropic mechanical properties of 2D materials with asymmetric in-plane lattices, such as black phosphorous, ${ }^{98}$ is much more challenging but will be very interesting. In 2D vertical heterostructures, interlayer interaction still needs to be quantified and understood. On the other hand, 2D planar heterostructures with a single atomic layer composed of two distinct materials attract much attention, but mechanical properties of these planar heterostructures are still unexplored. These topics will be particularly significant if the mechanical interactions in 2D heterostructures can be related to their electrical or optical properties.

Quantification of the relationship between defects and mechanical properties of 2D TMDs is also a question remained to be better answered. As 2D TMDs do not consist of a single type of atoms like graphene, the types of defects as well as their effects on mechanical properties of 2D TMDs would be more complicated. Unraveling these effects may yield lots of novel phenomena and enrich the understanding of defects in 2D TMDs. Particularly in 2D heterostructures, defects may enhance or weaken the interlayer interaction, and as a result, modulate the mechanical properties as well electrical, optical, and thermal properties of the system. These modulations provide new insights to the interlayer coupling, and will shed light on defect engineering of $2 \mathrm{D}$ heterostructures in general.

\section{ACKNOWLEDGMENTS}

J.W. acknowledges supports by the Office of Science, Office of Basic Energy Sciences, of the U.S. Department of Energy under Contract No. DE-AC02-05CH11231, and the NSF Center for Energy Efficient Electronics Science (NSF Award No. ECCS-0939514). K.L. acknowledges the support by "the Recruitment Program of Global Youth Experts (the Thousand Youth Talents Program)."

\section{REFERENCES}

1. M.M.J. Treacy, T.W. Ebbesen, and J.M. Gibson: Exceptionally high young's modulus observed for individual carbon nanotubes. Nature 381(6584), 678 (1996).

2. E.W. Wong, P.E. Sheehan, and C.M. Lieber: Nanobeam mechanics: Elasticity, strength, and toughness of nanorods and nanotubes. Science 277(5334), 1971 (1997).

3. C. Lee, X.D. Wei, J.W. Kysar, and J. Hone: Measurement of the elastic properties and intrinsic strength of monolayer graphene. Science 321(5887), 385 (2008). 
4. Y.J. Tian, B. Xu, D.L. Yu, Y.M. Ma, Y.B. Wang, Y.B. Jiang, W.T. Hu, C.C. Tang, Y.F. Gao, K. Luo, Z.S. Zhao, L.M. Wang, B. Wen, J.L. He, and Z.Y. Liu: Ultrahard nanotwinned cubic boron nitride. Nature 493(7432), 385 (2013).

5. U. Ozgur, Y.I. Alivov, C. Liu, A. Teke, M.A. Reshchikov, S. Dogan, V. Avrutin, S.J. Cho, and H. Morkoc: A comprehensive review of zno materials and devices. J. Appl. Phys. 98(4), 103 (2005).

6. J.Q. Wu: When group-iii nitrides go infrared: New properties and perspectives. J. Appl. Phys. 106(1), 011101 (2009).

7. Y.G. Sun and J.A. Rogers: Inorganic semiconductors for flexible electronics. Adv. Mater. 19(15), 1897 (2007).

8. J.A. Rogers, T. Someya, and Y.G. Huang: Materials and mechanics for stretchable electronics. Science 327(5973), 1603 (2010).

9. D.H. Kim, N.S. Lu, R. Ma, Y.S. Kim, R.H. Kim, S.D. Wang, J. Wu, S.M. Won, H. Tao, A. Islam, K.J. Yu, T.I. Kim, R. Chowdhury, M. Ying, L.Z. Xu, M. Li, H.J. Chung, H. Keum, M. McCormick, P. Liu, Y.W. Zhang, F.G. Omenetto, Y.G. Huang, T. Coleman, and J.A. Rogers: Epidermal electronics. Science 333(6044), 838 (2011).

10. H.W. Kroto, J.R. Heath, S.C. Obrien, R.F. Curl, and R.E. Smalley: C-60-buckminsterfullerene. Nature 318(6042), 162 (1985).

11. S. Iijima: Helical microtubules of graphitic carbon. Nature $\mathbf{3 5 4}$ (6348), 56 (1991).

12. A.P. Alivisatos: Semiconductor clusters, nanocrystals, and quantum dots. Science 271(5251), 933 (1996).

13. H.J. Dai: Carbon nanotubes: Synthesis, integration, and properties. Accounts Chem. Res. 35(12), 1035 (2002).

14. Y.N. Xia, P.D. Yang, Y.G. Sun, Y.Y. Wu, B. Mayers, B. Gates, Y.D. Yin, F. Kim, and Y.Q. Yan: One-dimensional nanostructures: Synthesis, characterization, and applications. Adv. Mater. 15 (5), 353 (2003).

15. K.S. Novoselov, A.K. Geim, S.V. Morozov, D. Jiang, Y. Zhang, S.V. Dubonos, I.V. Grigorieva, and A.A. Firsov: Electric field effect in atomically thin carbon films. Science 306(5696), 666 (2004).

16. K.S. Novoselov, D. Jiang, F. Schedin, T.J. Booth, V.V. Khotkevich, S.V. Morozov, and A.K. Geim: Two-dimensional atomic crystals. Proc. Natl. Acad. Sci. U. S. A. 102(30), 10451 (2005).

17. S.Z. Butler, S.M. Hollen, L.Y. Cao, Y. Cui, J.A. Gupta, H.R. Gutierrez, T.F. Heinz, S.S. Hong, J.X. Huang, A.F. Ismach, E. Johnston-Halperin, M. Kuno, V.V. Plashnitsa, R.D. Robinson, R.S. Ruoff, S. Salahuddin, J. Shan, L. Shi, M.G. Spencer, M. Terrones, W. Windl, and J.E. Goldberger: Progress, challenges, and opportunities in two-dimensional materials beyond graphene. ACS Nano 7(4), 2898 (2013).

18. K.S. Novoselov, A.K. Geim, S.V. Morozov, D. Jiang, M.I. Katsnelson, I.V. Grigorieva, S.V. Dubonos, and A.A. Firsov: Two-dimensional gas of massless dirac fermions in graphene. Nature 438(7065), 197 (2005).

19. K.F. Mak, C. Lee, J. Hone, J. Shan, and T.F. Heinz: Atomically thin $\mathrm{MoS}_{2}$ : A new direct-gap semiconductor. Phys. Rev. Lett. 105 (13), 4 (2010).

20. A. Splendiani, L. Sun, Y.B. Zhang, T.S. Li, J. Kim, C.Y. Chim, G. Galli, and F. Wang: Emerging photoluminescence in monolayer $\mathrm{MoS}_{2}$. Nano Lett. 10(4), 1271 (2010).

21. Y.B. Zhang, Y.W. Tan, H.L. Stormer, and P. Kim: Experimental observation of the quantum hall effect and berry's phase in graphene. Nature 438(7065), 201 (2005).

22. M. Liu, X.B. Yin, E. Ulin-Avila, B.S. Geng, T. Zentgraf, L. Ju, F. Wang, and X. Zhang: A graphene-based broadband optical modulator. Nature 474(7349), 64 (2011).

23. L. Ju, B.S. Geng, J. Horng, C. Girit, M. Martin, Z. Hao, H.A. Bechtel, X.G. Liang, A. Zettl, Y.R. Shen, and F. Wang: Graphene plasmonics for tunable terahertz metamaterials. Nat. Nanotechnol. 6(10), 630 (2011).
24. B. Radisavljevic, A. Radenovic, J. Brivio, V. Giacometti, and A. Kis: Single-layer $\mathrm{MoS}_{2}$ transistors. Nat. Nanotechnol. 6(3), 147 (2011).

25. D. Xiao, G.B. Liu, W.X. Feng, X.D. Xu, and W. Yao: Coupled spin and valley physics in monolayers of $\mathrm{MoS}_{2}$ and other group-vi dichalcogenides. Phys. Rev. Lett. 108(19), 5 (2012).

26. K.F. Mak, K.L. He, C. Lee, G.H. Lee, J. Hone, T.F. Heinz, and J. Shan: Tightly bound trions in monolayer $\mathrm{MoS}_{2}$. Nat. Mater. 12(3), 207 (2013).

27. Z.L. Ye, T. Cao, K. O'Brien, H.Y. Zhu, X.B. Yin, Y. Wang, S.G. Louie, and $X$. Zhang: Probing excitonic dark states in singlelayer tungsten disulphide. Nature 513(7517), 214 (2014).

28. J. Kim, X.P. Hong, C.H. Jin, S.F. Shi, C.Y.S. Chang, M.H. Chiu, L.J. Li, and F. Wang: Ultrafast generation of pseudo-magnetic field for valley excitons in $\mathrm{WSe}_{2}$ monolayers. Science 346(6214), 1205 (2014).

29. M.F. Yu, O. Lourie, M.J. Dyer, K. Moloni, T.F. Kelly, and R.S. Ruoff: Strength and breaking mechanism of multiwalled carbon nanotubes under tensile load. Science 287(5453), 637 (2000).

30. X.D. Han, K. Zheng, Y.F. Zhang, X.N. Zhang, Z. Zhang, and Z.L. Wang: Low-temperature in situ large-strain plasticity of silicon nanowires. Adv. Mater. 19(16), 2112 (2007).

31. Y. Zhu, F. Xu, Q.Q. Qin, W.Y. Fung, and W. Lu: Mechanical properties of vapor-liquid-solid synthesized silicon nanowires. Nano Lett. 9(11), 3934 (2009).

32. K.T. Wan, S. Guo, and D.A. Dillard: A theoretical and numerical study of a thin clamped circular film under an external load in the presence of a tensile residual stress. Thin Solid Films 425(1-2), 150 (2003)

33. U. Komaragiri, M.R. Begley, and J.G. Simmonds: The mechanical response of freestanding circular elastic films under point and pressure loads. J. Appl. Mech. 72(2), 203 (2005).

34. O.L. Blakslee: Elastic constants of compression-annealed pyrolytic graphite. J. Appl. Phys. 41(8), 3373 (1970).

35. H. Zhao, K. Min, and N.R. Aluru: Size and chirality dependent elastic properties of graphene nanoribbons under uniaxial tension. Nano Lett. 9(8), 3012 (2009).

36. G. Van Lier, C. Van Alsenoy, V. Van Doren, and P. Geerlings: Ab initio study of the elastic properties of single-walled carbon nanotubes and graphene. Chem. Phys. Lett. 326(1-2), 181 (2000)

37. F. Liu, P.M. Ming, and J. Li: Ab initio calculation of ideal strength and phonon instability of graphene under tension. Phys. Rev. B. 76(6), 7 (2007).

38. N.M. Bhatia and W. Nachbar: Finite indentation of elasticperfectly plastic membranes by a spherical indenter. Int. J. NonLinear Mech. 3(3), 307 (1968).

39. P.Y. Huang, C.S. Ruiz-Vargas, A.M. van der Zande, W.S. Whitney, M.P. Levendorf, J.W. Kevek, S. Garg, J.S. Alden, C.J. Hustedt, Y. Zhu, J. Park, P.L. McEuen, and D.A. Muller: Grains and grain boundaries in single-layer graphene atomic patchwork quilts. Nature 469(7330), 389 (2011).

40. R. Dettori, E. Cadelano, and L. Colombo: Elastic fields and moduli in defected graphene. J. Phys.: Condens. Matter 24(10), 10 (2012).

41. N.N. Jing, Q.Z. Xue, C.C. Ling, M.X. Shan, T. Zhang, X.Y. Zhou, and Z.Y. Jiao: Effect of defects on Young's modulus of graphene sheets: A molecular dynamics simulation. RSC Adv. 2(24), 9124 (2012).

42. R. Grantab, V.B. Shenoy, and R.S. Ruoff: Anomalous strength characteristics of tilt grain boundaries in graphene. Science 330(6006), 946 (2010).

43. J. Wei, J.T. Wu, H.Q. Yin, X.H. Shi, R.G. Yang, and M. Dresselhaus: The nature of strength enhancement and weakening by pentagon-heptagon defects in graphene. Nat. Mater. 11(9), 759 (2012) 
44. J.H. Warner, E.R. Margine, M. Mukai, A.W. Robertson, F. Giustino, and A.I. Kirkland: Dislocation-driven deformations in graphene. Science 337(6091), 209 (2012).

45. X.S. Li, W.W. Cai, J.H. An, S. Kim, J. Nah, D.X. Yang, R. Piner, A. Velamakanni, I. Jung, E. Tutuc, S.K. Banerjee, L. Colombo, and R.S. Ruoff: Large-area synthesis of high-quality and uniform graphene films on copper foils. Science 324(5932), 1312 (2009).

46. A. Reina, X.T. Jia, J. Ho, D. Nezich, H.B. Son, V. Bulovic, M.S. Dresselhaus, and J. Kong: Large area, few-layer graphene films on arbitrary substrates by chemical vapor deposition. Nano Lett. 9(1), 30 (2009).

47. G.H. Lee, R.C. Cooper, S.J. An, S. Lee, A. van der Zande, N. Petrone, A.G. Hammerherg, C. Lee, B. Crawford, W. Oliver, J.W. Kysar, and J. Hone: High-strength chemical-vapor deposited graphene and grain boundaries. Science 340(6136), 1073 (2013).

48. A.W. Tsen, L. Brown, M.P. Levendorf, F. Ghahari, P.Y. Huang, R.W. Havener, C.S. Ruiz-Vargas, D.A. Muller, P. Kim, and J. Park: Tailoring electrical transport across grain boundaries in polycrystalline graphene. Science 336(6085), 1143 (2012).

49. Q.K. Yu, L.A. Jauregui, W. Wu, R. Colby, J.F. Tian, Z.H. Su, H.L. Cao, Z.H. Liu, D. Pandey, D.G. Wei, T.F. Chung, P. Peng, N.P. Guisinger, E.A. Stach, J.M. Bao, S.S. Pei, and Y.P. Chen: Control and characterization of individual grains and grain boundaries in graphene grown by chemical vapour deposition. Nat. Mater. 10(6), 443 (2011).

50. K. Kim, Z. Lee, W. Regan, C. Kisielowski, M.F. Crommie, and A. Zettl: Grain boundary mapping in polycrystalline graphene. ACS Nano 5(3), 2142 (2011).

51. C.S. Ruiz-Vargas, H.L.L. Zhuang, P.Y. Huang, A.M. van der Zande, S. Garg, P.L. McEuen, D.A. Muller, R.G. Hennig, and J. Park: Softened elastic response and unzipping in chemical vapor deposition graphene membranes. Nano Lett. 11(6), 2259 (2011).

52. Q.Y. Lin, G. Jing, Y.B. Zhou, Y.F. Wang, J. Meng, Y.Q. Bie, D.P. Yu, and Z.M. Liao: Stretch-induced stiffness enhancement of graphene grown by chemical vapor deposition. ACS Nano 7(2), 1171 (2013).

53. A. Zandiatashbar, G.H. Lee, S.J. An, S. Lee, N. Mathew, M. Terrones, T. Hayashi, C.R. Picu, J. Hone, and N. Koratkar: Effect of defects on the intrinsic strength and stiffness of graphene. Nat. Commun. 5, 3186 (2014).

54. G. Lopez-Polin, C. Gomez-Navarro, V. Parente, F. Guinea, M.I. Katsnelson, F. Perez-Murano, and J. Gomez-Herrero: Increasing the elastic modulus of graphene by controlled defect creation. Nat. Phys. 11(1), 26 (2015).

55. M.M. Lucchese, F. Stavale, E.H.M. Ferreira, C. Vilani, M.V.O. Moutinho, R.B. Capaz, C.A. Achete, and A. Jorio: Quantifying ion-induced defects and raman relaxation length in graphene. Carbon 48(5), 1592 (2010).

56. L.G. Cancado, A. Jorio, E.H.M. Ferreira, F. Stavale, C.A. Achete, R.B. Capaz, M.V.O. Moutinho, A. Lombardo, T.S. Kulmala, and A.C. Ferrari: Quantifying defects in graphene via Raman spectroscopy at different excitation energies. Nano Lett. 11(8), 3190 (2011).

57. Y.W. Zhu, S. Murali, W.W. Cai, X.S. Li, J.W. Suk, J.R. Potts, and R.S. Ruoff: Graphene and graphene oxide: Synthesis, properties, and applications. Adv. Mater. 22(35), 3906 (2010).

58. K. Liu, C-L. Hsin, D. Fu, J. Suh, S. Tongay, M. Chen, Y. Sun, A. Yan, J. Park, K.M. Yu, W. Guo, A. Zettl, H. Zheng, D.C. Chrzan, and J. Wu: Self-passivation of defects: Effects of high-energy particle irradiation on elastic modulus of multilayer graphene. Adv. Mater. (2015), doi: 10.1002/adma.201501752.

59. A.K. Geim: Graphene: Status and prospects. Science 324(5934), 1530 (2009).

60. A.K. Geim and I.V. Grigorieva: van der Waals heterostructures. Nature 499(7459), 419 (2013).
61. O. Lopez-Sanchez, D. Lembke, M. Kayci, A. Radenovic, and A. Kis: Ultrasensitive photodetectors based on monolayer $\mathrm{MoS}_{2}$. Nat. Nanotechnol. 8(7), 497 (2013).

62. Q.H. Wang, K. Kalantar-Zadeh, A. Kis, J.N. Coleman, and M.S. Strano: Electronics and optoelectronics of two-dimensional transition metal dichalcogenides. Nature Nanotechnol. 7(11), 699 (2012).

63. Q.Y. He, Z.Y. Zeng, Z.Y. Yin, H. Li, S.X. Wu, X. Huang, and H. Zhang: Fabrication of flexible $\mathrm{MoS}_{2}$ thin-film transistor arrays for practical gas-sensing applications. Small 8(19), 2994 (2012).

64. J. Pu, Y. Yomogida, K.K. Liu, L.J. Li, Y. Iwasa, and T. Takenobu: Highly flexible $\mathrm{MoS}_{2}$ thin-film transistors with ion gel dielectrics. Nano Lett. 12(8), 4013 (2012).

65. H.Y. Chang, S.X. Yang, J.H. Lee, L. Tao, W.S. Hwang, D. Jena, N.S. Lu, and D. Akinwande: High-performance, highly bendable $\mathrm{MoS}_{2}$ transistors with high-k dielectrics for flexible low-power systems. ACS Nano 7(6), 5446 (2013).

66. D. Akinwande, N. Petrone, and J. Hone: Two-dimensional flexible nanoelectronics. Nat. Commun. 5, 12 (2014).

67. S. Bertolazzi, J. Brivio, and A. Kis: Stretching and breaking of ultrathin $\mathrm{MoS}_{2}$. ACS Nano 5(12), 9703 (2011).

68. A. Castellanos-Gomez, M. Poot, G.A. Steele, H.S.J. van der Zant, N. Agrait, and G. Rubio-Bollinger: Elastic properties of freely suspended $\mathrm{MoS}_{2}$ nanosheets. Adv. Mater. 24(6), 772 (2012).

69. J.L. Feldman: Elastic-constants of $2 \mathrm{~h}-\mathrm{MoS}_{2}$ and $2 \mathrm{~h}-\mathrm{NbSe}_{2}$ extracted from measured dispersion curves and linear compressibilities. J. Phys. Chem. Solids 37(12), 1141 (1976).

70. Y.H. Lee, X.Q. Zhang, W.J. Zhang, M.T. Chang, C.T. Lin, K.D. Chang, Y.C. Yu, J.T.W. Wang, C.S. Chang, L.J. Li, and T.W. Lin: Synthesis of large-area $\mathrm{MoS}_{2}$ atomic layers with chemical vapor deposition. Adv. Mater. 24(17), 2320 (2012).

71. K.K. Liu, W.J. Zhang, Y.H. Lee, Y.C. Lin, M.T. Chang, C. Su, C.S. Chang, H. Li, Y.M. Shi, H. Zhang, C.S. Lai, and L.J. Li Growth of large-area and highly crystalline $\mathrm{MoS}_{2}$ thin layers on insulating substrates. Nano Lett. 12(3), 1538 (2012).

72. S. Najmaei, Z. Liu, W. Zhou, X.L. Zou, G. Shi, S.D. Lei, B.I. Yakobson, J.C. Idrobo, P.M. Ajayan, and J. Lou: Vapour phase growth and grain boundary structure of molybdenum disulphide atomic layers. Nat. Mater. 12(8), 754 (2013).

73. A.M. van der Zande, P.Y. Huang, D.A. Chenet, T.C. Berkelbach, Y.M. You, G.H. Lee, T.F. Heinz, D.R. Reichman, D.A. Muller, and J.C. Hone: Grains and grain boundaries in highly crystalline monolayer molybdenum disulphide. Nat. Mater. 12(6), 554 (2013).

74. K. Kang, S.E. Xie, L.J. Huang, Y.M. Han, P.Y. Huang, K.F. Mak, C.J. Kim, D. Muller, and J. Park: High-mobility three-atom-thick semiconducting films with wafer-scale homogeneity. Nature 520(7549), 656 (2015).

75. K. Liu, Q.M. Yan, M. Chen, W. Fan, Y.H. Sun, J. Suh, D.Y. Fu, S. Lee, J. Zhou, S. Tongay, J. Ji, J.B. Neaton, and J.Q. Wu: Elastic properties of chemical-vapor-deposited monolayer $\mathrm{MoS}_{2}, \mathrm{WS}_{2}$, and their bilayer heterostructures. Nano Lett. 14(9), 5097 (2014).

76. C. Filippi, D.J. Singh, and C.J. Umrigar: All-electron local-density and generalized-gradient calculations of the structural-properties of semiconductors. Phys. Rev. B. 50(20), 14947 (1994).

77. J. Kang, S. Tongay, J. Zhou, J.B. Li, and J.Q. Wu: Band offsets and heterostructures of two-dimensional semiconductors. Appl. Phys. Lett. 102(1), 4 (2013).

78. W.J. Yu, Z. Li, H.L. Zhou, Y. Chen, Y. Wang, Y. Huang, and X.F. Duan: Vertically stacked multi-heterostructures of layered materials for logic transistors and complementary inverters. Nat. Mater. 12(3), 246 (2013).

79. W.J. Yu, Y. Liu, H.L. Zhou, A.X. Yin, Z. Li, Y. Huang, and X.F. Duan: Highly efficient gate-tunable photocurrent generation in vertical heterostructures of layered materials. Nat. Nanotechnol. 8(12), 952 (2013). 
80. L. Britnell, R.M. Ribeiro, A. Eckmann, R. Jalil, B.D. Belle, A. Mishchenko, Y.J. Kim, R.V. Gorbachev, T. Georgiou, S.V. Morozov, A.N. Grigorenko, A.K. Geim, C. Casiraghi, A.H. Castro Neto, and K.S. Novoselov: Strong light-matter interactions in heterostructures of atomically thin films. Science 340(6138), 1311 (2013).

81. B. Hunt, J.D. Sanchez-Yamagishi, A.F. Young, M. Yankowitz, B.J. LeRoy, K. Watanabe, T. Taniguchi, P. Moon, M. Koshino, P. Jarillo-Herrero, and R.C. Ashoori: Massive dirac fermions and hofstadter butterfly in a van der Waals heterostructure. Science 340(6139), 1427 (2013).

82. S. Tongay, W. Fan, J. Kang, J. Park, U. Koldemir, J. Suh, D.S. Narang, K. Liu, J. Ji, J.B. Li, R. Sinclair, and J.Q. Wu: Tuning interlayer coupling in large-area heterostructures with codgrown $\mathrm{MoS}_{2}$ and $\mathrm{WS}_{2}$ monolayers. Nano Lett. 14(6), 3185 (2014).

83. X.P. Hong, J. Kim, S.F. Shi, Y. Zhang, C.H. Jin, Y.H. Sun, S. Tongay, J.Q. Wu, Y.F. Zhang, and F. Wang: Ultrafast charge transfer in atomically thin $\mathrm{MoS}_{2} / \mathrm{WS}_{2}$ heterostructures. Nat. Nanotechnol. 9(9), 682 (2014).

84. Y.J. Gong, J.H. Lin, X.L. Wang, G. Shi, S.D. Lei, Z. Lin, X.L. Zou, G.L. Ye, R. Vajtai, B.I. Yakobson, H. Terrones, M. Terrones, B.K. Tay, J. Lou, S.T. Pantelides, Z. Liu, W. Zhou, and P.M. Ajayan: Vertical and in-plane heterostructures from $\mathrm{WS}_{2} / \mathrm{MoS}_{2}$ monolayers. Nat. Mater. 13(12), 1135 (2014).

85. C.M. Huang, S.F. Wu, A.M. Sanchez, J.J.P. Peters, R. Beanland, J.S. Ross, P. Rivera, W. Yao, D.H. Cobden, and X.D. Xu: Lateral heterojunctions within monolayer $\mathrm{MoSe}_{2}-\mathrm{WSe}_{2}$ semiconductors. Nat. Mater. 13(12), 1096 (2014).

86. K.H. Liu, L.M. Zhang, T. Cao, C.H. Jin, D.A. Qiu, Q. Zhou, A. Zettl, P.D. Yang, S.G. Louie, and F. Wang: Evolution of interlayer coupling in twisted molybdenum disulfide bilayers. Nat. Commun. 5, 6 (2014).

87. P.H. Tan, W.P. Han, W.J. Zhao, Z.H. Wu, K. Chang, H. Wang, Y.F. Wang, N. Bonini, N. Marzari, N. Pugno, G. Savini, A. Lombardo, and A.C. Ferrari: The shear mode of multilayer graphene. Nat. Mater. 11(4), 294 (2012).
88. J.B. Wu, X. Zhang, M. Ijas, W.P. Han, X.F. Qiao, X.L. Li, D.S. Jiang, A.C. Ferrari, and P.H. Tan: Resonant raman spectroscopy of twisted multilayer graphene. Nat. Commun. 5, 8 (2014).

89. E. Koren, E. Lortscher, C. Rawlings, A.W. Knoll, and U. Duerig: Adhesion and friction in mesoscopic graphite contacts. Science 348(6235), 679 (2015).

90. H.G. Craighead: Nanoelectromechanical systems. Science 290(5496), 1532 (2000).

91. J.S. Bunch, A.M. van der Zande, S.S. Verbridge, I.W. Frank, D.M. Tanenbaum, J.M. Parpia, H.G. Craighead, and P.L. McEuen: Electromechanical resonators from graphene sheets. Science 315(5811), 490 (2007).

92. J.T. Robinson, M. Zalalutdinov, J.W. Baldwin, E.S. Snow, Z.Q. Wei, P. Sheehan, and B.H. Houston: Wafer-scale reduced graphene oxide films for nanomechanical devices. Nano Lett. 8(10), 3441 (2008).

93. C.Y. Chen, S. Rosenblatt, K.I. Bolotin, W. Kalb, P. Kim, I. Kymissis, H.L. Stormer, T.F. Heinz, and J. Hone: Performance of monolayer graphene nanomechanical resonators with electrical readout. Nat. Nanotechnol. 4(12), 861 (2009).

94. J.T. Robinson, M.K. Zalalutdinov, C.E. Junkermeier, J.C. Culbertson, T.L. Reinecke, R. Stine, P.E. Sheehan, B.H. Houston, and E.S. Snow: Structural transformations in chemically modified graphene. Solid State Commun. 152(21), 1990 (2012).

95. A. Eichler, J. Moser, J. Chaste, M. Zdrojek, I. Wilson-Rae, and A. Bachtold: Nonlinear damping in mechanical resonators made from carbon nanotubes and graphene. Nat. Nanotechnol. 6(6), 339 (2011).

96. A. Castellanos-Gomez, R. van Leeuwen, M. Buscema, H.S.J. van der Zant, G.A. Steele, and W.J. Venstra: Single-layer $\mathrm{MoS}_{2}$ mechanical resonators. Adv. Mater. 25(46), 6719 (2013).

97. J. Lee, Z.H. Wang, K.L. He, J. Shan, and P.X.L. Feng: High frequency $\mathrm{MoS}_{2}$ nanomechanical resonators. ACS Nano 7(7), 6086 (2013).

98. L.K. Li, Y.J. Yu, G.J. Ye, Q.Q. Ge, X.D. Ou, H. Wu, D.L. Feng, X.H. Chen, and Y.B. Zhang: Black phosphorus field-effect transistors. Nat. Nanotechnol. 9(5), 372 (2014). 